\title{
Agua y cereal: los molinos hidráulicos en Arroyomolinos de León
}

Gema Carrera Díaz, Aniceto Delgado Méndez, Pilar Zafra Costán. Área de Etnología. Centro de Documentación del IAPH

La escasez de agua de

sus arroyos

condiciona las formas

de los elementos

constructivos de

conducción,

almacenamiento

y molienda
A lo largo de esta sección sobre la Sierra de Aracena y Picos de Aroche son numerosos los bienes descritos cuyo nexo de unión se establece a partir de las diferentes formas de captar, almacenar y distribuir el agua.

Entre los diversos ejemplos localizables en este amplio territorio destacan por su número y diversidad dependiendo del contexto hidráulico, los molinos de agua, también conocidos como de rodezno o harineros. Situados en las inmediaciones de riveras y arroyos, los molinos aparecen en la mayoría de las poblaciones que conforman esta comarca serrana, siendo fácilmente visibles en Alájar, Linares de la Sierra, Encinasola, Cañaveral de León o Aroche, entre otras localidades.

Aunque se desconoce el número exacto de molinos de agua que han existido en esta comarca, parece ser que a finales del siglo XIX eran más de cien los que estaban en funcionamiento.

Una de las localidades donde la actividad de la molienda adquirió una especial relevancia fue Arroyomolinos de León, tal y como se desprende de su etimología y del significado que estos bienes han tenido para sus vecinos.
A pesar de la escasa documentación acerca del origen de estos inmuebles y de la actividad en ellos desarrollada, encontramos las primeras referencias en el Diccionario geográfico-estadístico-histórico de Pascual Madoz (18451850) cuando nombra al municipio de Arroyomolinos de León y describe que "además de proporcionar el riego necesario, las aguas del arroyo de la Cala y el de la Garganta, dan impulso a 18 molinos harineros de poca cantidad, ya por la escasez de aquellas para este efecto, ya por ser de pozo o cubo". El elevado número de estos bienes nos muestra la importancia que tuvo la actividad de la molienda en este lugar y el alto grado de especialización de su población, dando respuesta a la llegada de grandes cantidades de cereal procedentes no solamente de localidades vecinas sino también de otros pueblos de Portugal y de la cercana Extremadura. La escasez de agua de sus arroyos es condicionante de las formas que adquieren los elementos constructivos de conducción, almacenamiento y sistemas de molienda en la zona.

Dependiendo de la orografía y del caudal de la red hidrográfica, el molino tenía unas características arquitectónicas u otras, de ahí que el conocimiento del terreno se convirtiera en uno de los principales requisitos de los 


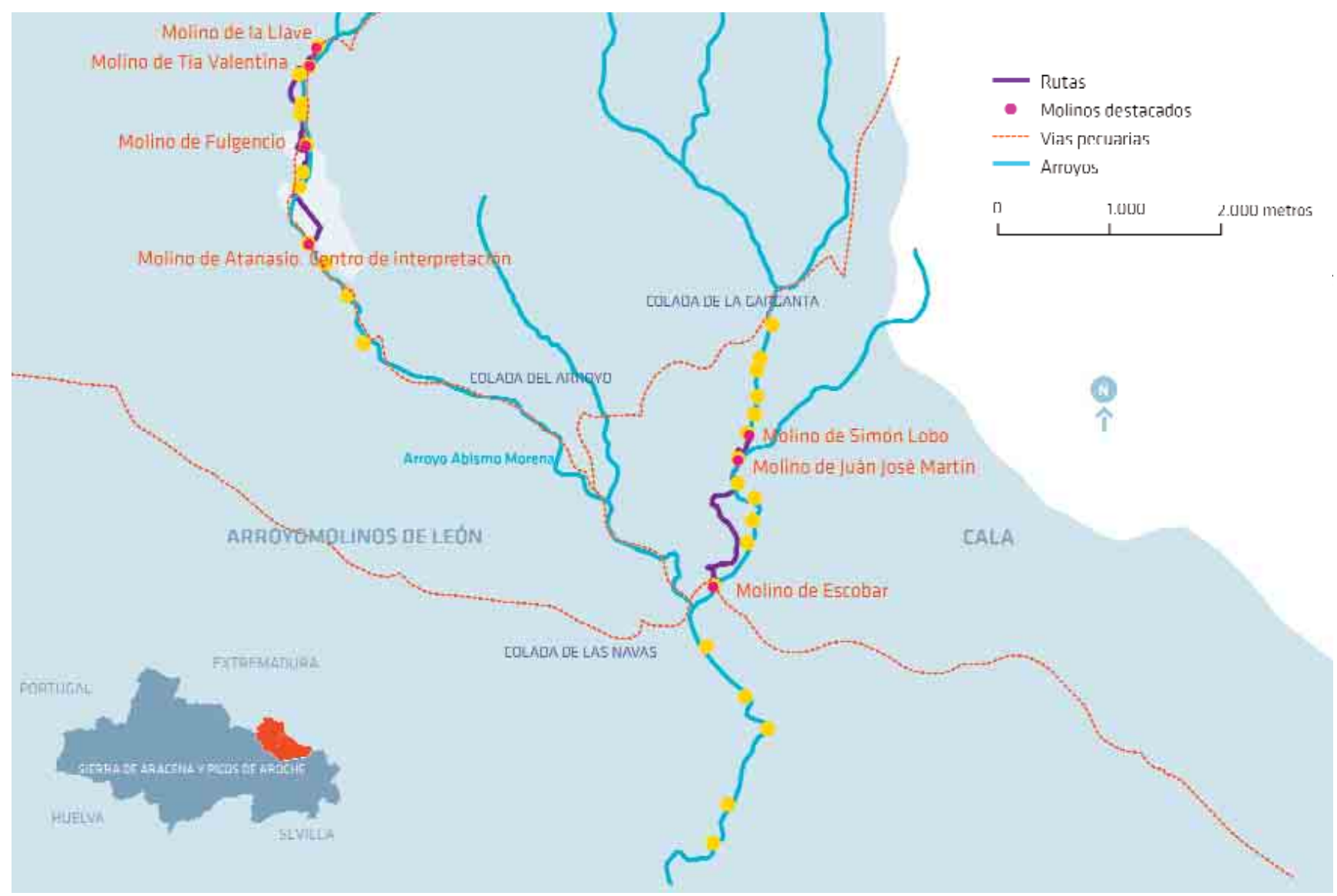

(1) Recorrido por los molinos hidráulicos de Arroyomolinos de León / GEMA CARRERA, DAVID VILLALóN, IAPH

alarifes para aplicar las técnicas constructivas más apropiadas al medio. Además de la localización de estos bienes junto a los arroyos y debido al poco caudal de los mismos, un sistema de largas acequias, presas y represas permitía distribuir y acumular el agua para su óptimo aprovechamiento. En Arroyomolinos de León, la mayoría de molinos emplearon los desniveles del terreno para optimizar la fuerza del agua y su escasez, sobre todo en época estival.

Esta deficiencia de agua hacía necesaria la construcción de represas para su almacenamiento. Elemento que daba nombre al sistema de molienda: moler de represa frente al moler a hilo en ríos de mayor caudal y corriente continua. Esto condicionaba también el sistema y organización del trabajo de los molinos ya que debía finalizar su labor el primero, "Molino de la Llave", para que pudieran moler los sucesivos. Cuando la presa de este molino acumulaba agua suficiente, comenzaba el proceso $y$, tras la molienda, dejaba correr el agua para que fuera almacenada en la presa del siguiente molino. Este complejo y laborioso proceso permitía aprovechar el agua del momento, estableciendo a su vez determinados horarios a los que cada molinero debía adaptarse
Otro hecho significativo de los molinos de agua en la Sierra de Huelva es su diversidad respecto al léxico de la actividad y los materiales y herramientas utilizados durante el proceso de molturación del cereal. Esta variedad genera una enorme riqueza poco constatada en las investigaciones y estudios realizados.

Tras diferentes momentos de esplendor, los avatares de la guerra civil trajeron como consecuencia la crisis de la actividad y su desaparición en los años sesenta. La prohibición, una vez finalizada la contienda, de utilizar los molinos provocó el abandono de la gran mayoría. A esto último contribuyó la creación de una Fábrica de Harina en Arroyomolinos a principios del siglo pasado, así como la pérdida de competitividad de los molinos respecto a las cantidades, calidades y beneficios de la producción industrial.

En este proceso de crisis de la actividad molinera han de señalarse diferentes aspectos de enorme interés para comprender su evolución y situación actual. A la pérdida de los conocimientos y saberes asociados a esta labor deberíamos unir el abandono de las propias instalaciones y su desaparición en algunos casos; el desprestigio sufrido por aquellas personas, que tenían en el trabajo del molino un ingreso más que unir a otras actividades fundamentalmente agroganaderas; y la aparición de estrategias (estraperlo) que intentarán mantener la actividad a pesar de su prohibición, debido a la escasez y necesidad de consumir pan.

En la actualidad, aunque los molinos han perdido sus funciones principales, constituyen un texto fundamental para conocer este territorio y comprender las transformaciones socioeconómicas de esta localidad serrana. Lejos de quedar en el olvido, los molinos se convierten en una de las principales señas de identidad de Arroyomolinos de León, tal y como ponen de manifiesto las diferentes estrategias seguidas para su puesta en valor y difusión.

Como medida para proceder a la protección de estos inmuebles, la Delegación Provincial de Cultura de Huelva realizó la documentación técnica para su inscripción en el Catálogo General del Patrimonio Histórico Andaluz. Posteriormente, la administración cultural autonómica, con la colaboración del Ayuntamiento de Arroyomolinos de León y de la asociación Alma Natura, llevaron a cabo la rehabilitación de un molino que en la actualidad es un Centro de Interpretación que pretende 


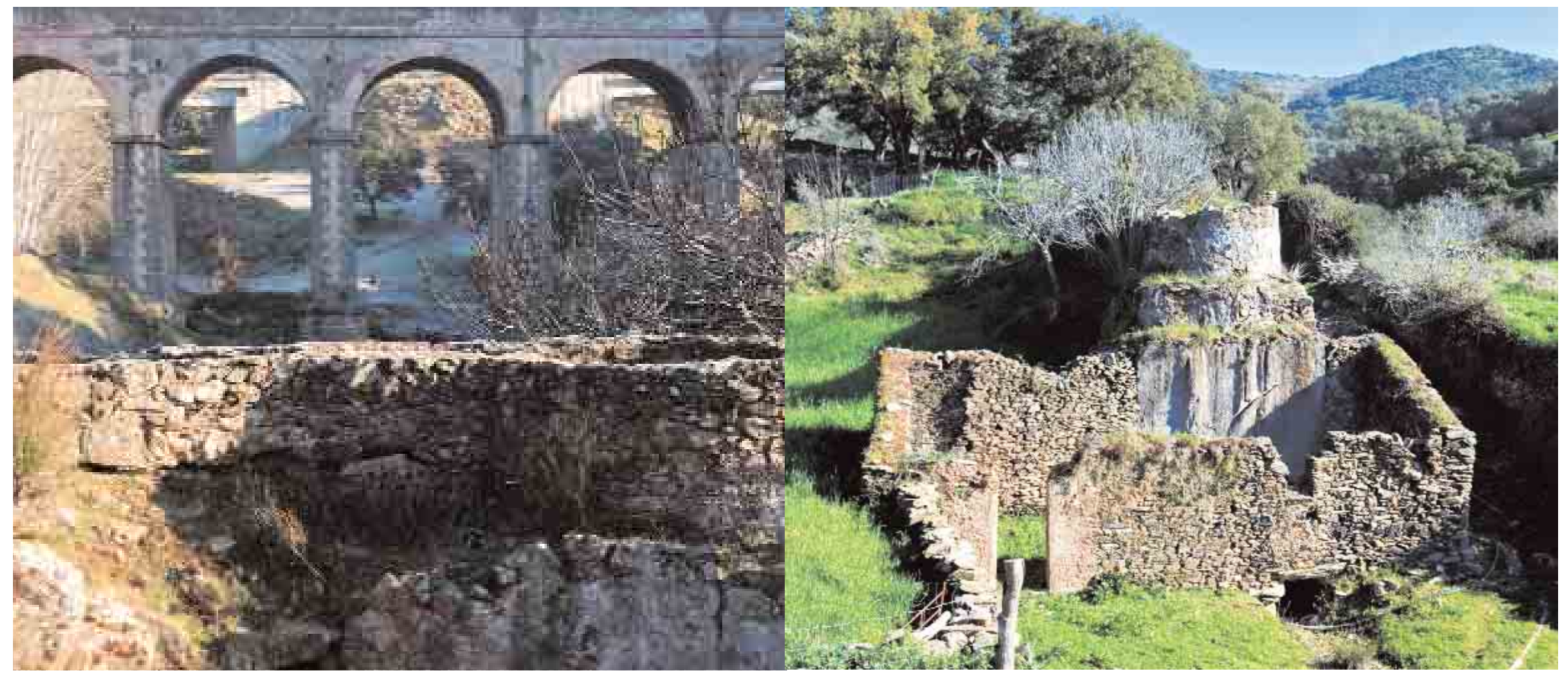

(1) Molino y Puente de la Gitana / AnICEto Delgado, IAPH

(1) Molino de la llave / JAVIER Romero, IAPH

Se desconoce el

número exacto de

molinos de agua que

han existido en la

comarca, pero a

finales del s. XIX

estaban en

funcionamiento más

de cien acercarse a estos inmuebles y al conocimiento de la actividad en ellos desarrollada.

Ruta de los molinos

Los ejes fluviales de los arroyos del AbismoMorena y el de Valdelamadera vertebran el recorrido que se presenta a continuación con el objetivo de favorecer el acercamiento a los diferentes bienes relacionados con el aprovechamiento del agua y la transformación del cereal en harina.

Aparejados a estos hitos patrimoniales, perviven numerosos testimonios materiales que ejemplifican las distintas formas de adaptación del hombre a su entorno. Proceso que ha generado un paisaje donde la canalización del agua se plasma en la interesante red de acequias y presas que jalonan los caminos donde transitan pasado y presente. Por ello, aún cuando el objetivo principal de esta ruta son los molinos de agua, habrá que hacer hincapié en la importancia que tienen para el conocimiento de este territorio cortijos, eras y pajares, cercas de piedra, huertos situados en los alrededores del municipio y demás elementos, pues resultan claves para la contextualización del itinerario elegido.

Antes de iniciar la andadura, conocer el Centro de Interpretación se torna indispensable. Situado a escasos metros de la población, permite obtener información sobre algunos de los aspectos más sobresalientes de esta ruta. Durante el recorrido, el visitante podrá visualizar, además de un plano situacional sobre los molinos, un montaje audiovisual donde se describen las partes que los conformaban y la actividad que en ellos se desarrollaba.

El punto de partida de esta ruta lo establece el molino de la Llave, apelativo que recibió por ser el primero en recoger el agua y aprovechar su fuerza para la molturación. Conocido tam- 


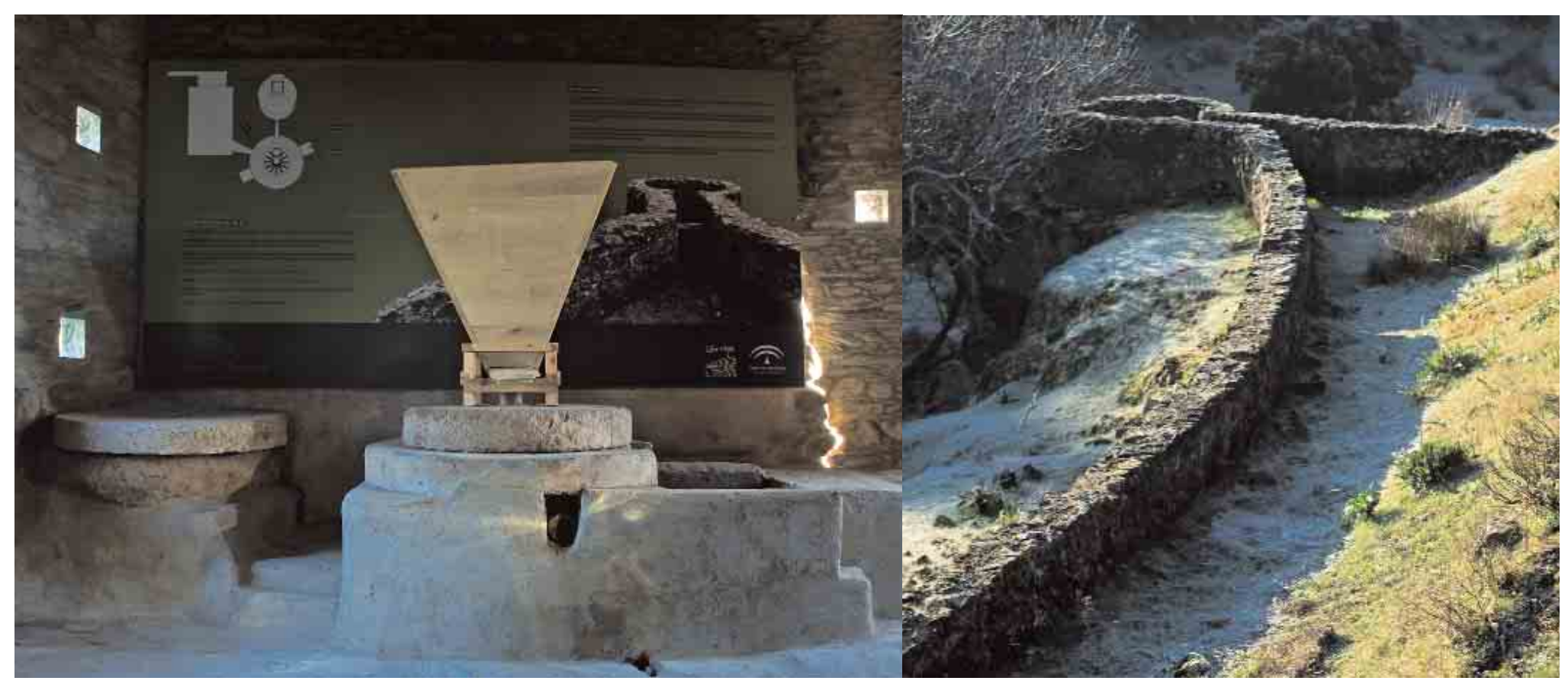

(1) Interior de la sala de la molienda del Molino de Atanasio, actual centro de interpretación / Javier Romero, IAPH

(1) Molino Juan José Martín / JAVIER Romero, IAPH

bién como molino de Blas Lozano, este inmueble estuvo en activo hasta los años sesenta $y$, como aún puede apreciarse, conserva parte de la sala de molienda. El paseo hasta este enclave permitirá ubicar el emplazamiento del municipio serrano de Arroyomolinos y observar el valle por el que se desliza el arroyo del Abismo-Morena.

Aunque no conserva la sala de molienda, el molino de Tía Valentina (los nombres que reciben estos inmuebles guardan una estrecha relación con las personas que fueron sus últimos propietarios) destaca por las dimensiones y el buen estado de conservación de su cubo y cítara.

Ya dentro del poblado podemos visitar varios molinos entre los que destacan el del Tío Fulgencio, que conserva en muy buen estado la cavucera, que recogía y transportaba el agua del arroyo hasta el molino, y la presa que culmina su construcción en el cubo.

También en los alrededores del pueblo pode-

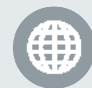

\section{En la web}

Grupo de Desarrollo Rural Sierra de Aracena y Picos de Aroche www.gdrsaypa.org

Asociación en la que están presentes más de 60 entidades interesadas en participar en el desarrollo de la comarca. Partiendo de las posibilidades y recursos de la zona, pretende favorecer la diversificación económica, la generación de empleo, la mejora de la calidad de vida de sus habitantes, así como facilitar la capacidad de organización de la población serrana y la articulación del territorio.

\section{Alma Natura}

www.almanatura.org

Con sede en Arroyomolinos de León, cuenta entre sus actividades con recorridos a través de las rutas patrimoniales diseñadas sobre los Molinos de Agua de la zona.

Ayuntamiento de Arroyomolinos de León

www.arroyomolinosdeleon.com

Este Ayuntamiento apuesta por el turismo rural y todo lo que genera a su alrededor como motor de desarrollo. La página web se plantea como una herramienta para dar a conocer la riqueza cultural y etnológica de la localidad. 
Bibliografía de cultura del agua en la Sierra de Huelva

mos observar el molino de Atanasio, inmueble que ha sido recientemente restaurado y convertido en el Centro de Interpretación de los molinos de esta localidad.

En pleno arroyo de Valdelamadera, ubicados en las proximidades del puente de la Gitana, se localizan los molinos de Simón Lobo y el de Juan José Martín. El primero de ellos fue el último en funcionar. Además, en los años sesenta, cuando cesó su producción, se intentó convertir este inmueble en un generador eléctrico pero debido a la poca velocidad que producía el agua no se obtuvo el resultado esperado

Por último, y no por ello menos importante, concluye la ruta en el molino de Escobar, uno de los ejemplos más significativos de esta tipología constructiva debido, entre otras razones, al buen estado de conservación que presenta tanto el cubo como la sala de molienda. Además, otro elemento de interés es la existencia de la casa donde vivía el molinero que se encuentra anexa al molino.

Agua y molino, cereal y piedra, forman una combinación que habla de experiencias compartidas, de procesos de adaptación que han confeccionado un texto donde los molinos representan un tiempo y un espacio para comprender formas y modos de vida de este territorio serrano.
CANTERo MARTíN, P.A. Formas de agua (a propósito del agua en Galaroza). Aestuaria. Revista de Investigación, $n^{\circ}$ 2, 1994, Diputación Provincial de Huelva, p. 68-85.

CANTERO MARTÍN, P.A. Los Jarritos. Fiesta del agua. El Fol-Klore andaluz, $n^{0} 7$ (1991).

CANTERO MARTÍN, P.A. Los Molinos de agua. Las riberas de Arroyomolinos de León. En Márquez Domínguez, J.A. (dir.) : Artes, costumbres y riquezas de la provincia de Huelva, tomo I. Madrid : Mediterráneo, 1997, p. 389-404

CANTERO MARTíN, P.A. Las moradas del agua. Arquitectura del agua en la provincia de Huelva. Narria : estudios de artes y costumbres populares, $n^{\circ} 81-84,1998$, p. 17-32 *

CANTERO MARTÍN, P.A. Resembrar el agua. En : Culturas del agua. Murcia : Consejería de Educación y Cultura, 2004, p. 251-271*

CARO BAROJA, J. La estación de amor : fiestas populares de Mayo a San Juan. Barcelona : Círculo de Lectores, 1992

DELGADO MÉNDEZ, A. La protección y difusión del patrimonio etnológico: arquitecturas de agua en la Sierra de Aracena y Picos de Aroche. En: Actas XXI Jornadas de Patrimonio de la Sierra. Huelva : Diputación Provincial de Huelva, 2007*

FAJARDO DE LA FUENTE, A.; TARÍN ALCALÁ-ZAMORA, A. Sierra de Aracena y Picos de Aroche : recorrido natural y cultural. Sevilla : Miguel Ángel Marín, 1999 *

GONZÁLEZ FLORES, S. La arquitectura del agua en Zufre, pilares y lavaderos. En : Patrimonio cultural de la provincia de Huelva : actas XV Jornadas del Patrimonio de la comarca de la Sierra. Aroche (Huelva), 13, 14, 15 y 16 de abril de 2000. Huelva : Diputación Provincial de Huelva, 2001, p. $433-442$ *

MACÍAS RICO, J.L. La fuente antigua o del Carmen en Galaroza. Otro ejemplo de fuente pública de topología medieval en la Sierra. En : Actas XIV Jornadas del Patrimonio de la comarca de la Sierra. Santa Ana la Real (Huelva), marzo de 1999. Huelva : Diputación Provincial de Huelva, 2000, p. $407-425$ *

MEDIANERO HERNÁNDEZ, J.M. Fuentes y lavaderos en la provincia de Huelva. Huelva Diputación Provincial de Huelva, 2003 *

MEDIANERO HERNÁNDEZ, J.M. (2004) "Mensajeros de Agua" en Fajardo, Antonio y Tarín, Amalia. Sierra de Aracena y Picos de Aroche. Recorrido Natural y Cultural. Sevilla, 2004

MUÑIZ CARRASCO, J.A. Tipología de molinos harineros en la Sierra. En : Ponencias y comunicaciones de las VI Jornadas del Patrimonio de la Sierra, Fuenteheridos (Huelva), marzo de 1991. Huelva : Diputación Provincial de Huelva, 1996, p. 93-115*

OJEDA RIVER, J.F.; SILVA PÉREZ, R. Aproximación a los paisajes de la Sierra Morena Andaluza. En : Paisaje y Ordenación del Territorio. Sevilla : Consejería de Obras Públicas y Transportes, 2002, p. $71-91^{*}$

OLIVER, A.; PLEGUEZUELO, A.; SÁNCHEZ, J.M. Guia histórico-artistica de la Sierra de Aracena y Picos de Aroche. Aracena : Iniciativas Leader Sierra de Aracena y Picos de Aroche, 2004 *

SÁNCHEZ Y SÁNCHEZ, J.M. Manuel Pérez y González : un proyecto de fuente monumental en Cortegana a finales del siglo XIX. En : Actas delas VI Jornadas del Patrimonio de la Sierra [de Huelva] : Fuenteheridos (Huelva) : 22, 23, 24 y 25 de marzo de 1991. Huelva : Diputación Provincial de Huelva, 1996, p.63-70*

Nota: Bibliografía resumida de la sección. Contiene referencias específicas asi como bibliografía citada expresamente por los autores. Para una mayor información puede dirigirse a a Biblioteca del IAPH. Las publicaciones marcadas con asterisco ${ }^{*}$ ) se encuentran disponibles para su consulta en la Biblioteca del IAPH. 
